\title{
Mathematical and simulation of lid driven cavity flow at different aspect ratios using single relaxation time lattice boltzmann technique
}

\author{
Anil Kumar ${ }^{1}$, S P Agrawal ${ }^{2}$ \\ ${ }^{1}$ Department of Applied Mathematics, World Institute of Technology Sohna Gurgaon, India \\ ${ }^{2}$ Department of Civil Engineering, World Institute of Technology Sohna Gurgaon, India \\ Email address: \\ dranilkumar73@rediffmail.com(A. Kumar)
}

\section{To cite this article:}

Anil Kumar, S P Agrawal. Mathematical and Simulation of Lid Driven Cavity Flow at Different Aspect Ratios Using Single Relaxation Time Lattice Boltzmann Technique. American Journal of Theoretical and Applied Statistics. Vol. 2, No. 3, 2013, pp. 87-93.

doi: $10.11648 /$ j.ajtas.20130203.17

\begin{abstract}
In this paper we, consider a restrictions on the choice of relaxation time in single relaxation time (SRT) models, simulation of flows is generally limited base on this technique. In the current study of the SRT lattice Boltzmann equation have been used to simulate lid driven cavity flow at various Reynolds numbers (100-5000) and three aspect ratios, $\mathrm{K}=1,1.5$ and 4. The point which is vital in convergence of this technique is how the boundary conditions will be implemented. Two kinds of boundary conditions which imply no-slip and constant inlet velocity, imposed in the present work. For square cavity, results show that with increasing the Reynolds number, bottom corner vortices will grow but they won't merge together. In this case which the aspect ratio equals four, and Reynolds number reaches over 1000, simulations predicted four primary vortices, which have not predicted by previous single relaxation time models. The results have been compared by previous multi relaxation model.
\end{abstract}

Keywords: Cavity Flow, Lattice Boltzmann, Aspect Ratio, Vortex Integration

\section{Introduction}

In recent decays, the Lattice Boltzmann equation has achieved an important role in solution of engineering problems. This fact is confirmed with numerous papers which have been published in recent years[1]. LBE with Bhatnagar-Gross-krook approximation (LBGK) model has reached the vast successes in fluid simulations. Using this model has many advantages such as application of boundary condition in complex geometries, reduction of running time and simple parallelism in computations[2-5]. But this model suffers from some numerical instabilities and limitations of usage of high Reynolds numbers. This problems cause that in some cases multiple-relaxation-time model be considered. Although this method increases the stability and limitation of the solution domain but simplicity of single relaxation time is the reason to more usage of this model. Solution of cavity flow has been studied by many people. Hou et al[6] and Guo et al[7] have studied cavity flow by LBGK model. Ghia[8] investigated this problem by MRT model which in this model collision term in generally is different with
LBGK. Etroke et al[9] completely studied cavity flow problem in high Reynolds numbers with stream function and vorticity formulation method. Taneda investigated the effect of aspect ratio on the laminar regime experimentally[10], and results have been verified numerically by Shen and Floryan[11].

By increasing the cavity depth, bottom corner vortices begin to grow and finally they merge and make another primary vortex. With further increases in cavity depth, two another bottom vortexes are created. This process is continuing as the cavity depth is increasing. Patil et al[12] has simulated the cavity flow with LBGK model in different Reynolds number ranges from 50 until 3200, and different aspect ratios between $\mathrm{K}=1$ and $\mathrm{K}=4$. Their conclusions were Compatible with Taneda and Chen works results. Few studies have conducted in cavity flow problem with Reynolds number more than 3200 and aspect ratio beyond 1 . Lin et al[14] has simulated the deep lid driven cavity flow in Reynolds numbers between 100 and 7500 and aspect ratios $\mathrm{K}=1$, 1.5, 4. They used Multi relaxation time lattice Boltzmann model and compared results with pervious 
works.

In this paper LBGK model is used for deep lid driven cavity flow simulation in Reynolds number ranges between 100 and 5000, and aspect ratios equal with $1,1.5$ and 4 , which is not used before and results are compared with latest results.

\section{Boltzmann Equation with BGK Approximation}

Consider a Lattice Boltzmann equation are given below (1)

$$
f_{i}\left(\vec{x}+c_{i} \delta t, t+\delta t\right)-f_{i}(\vec{x}, t)=\Omega_{i}
$$

Where $f_{i}$ is the distribution function for the particles which have discrete velocities indicated by $c_{i}$. Right hand side of the above equation includes the collision term and Bhatnagar-Gross-krook (BGK) approximation is used for evaluate this term by the following form:

$$
\Omega_{i}=-\frac{f_{i}-f_{i}^{e q}}{\tau}+\delta_{i} F_{i}
$$

In equation (2) $\tau$ is the relaxation time, $f_{i}^{e q}$ is the equilibrium distribution function and $\delta_{i} F$ denotes the external forces field. As introduced in[1] the equilibrium distribution function, $f_{i}^{e q}$ is evaluated by:

$$
f_{i}^{e q}=\rho \omega_{i}\left[1+\frac{3}{c_{s}^{2}} \vec{c} \cdot \vec{u}+\frac{9}{2 c_{s}^{4}}(\vec{c} \cdot \vec{u})^{2}-\frac{3}{2 c_{s}^{2}} \vec{u} \cdot \vec{u}\right]
$$

In equation (3) $u$ and $\rho$ are macroscopic velocity and density, respectively. $\omega_{i}$ and $c_{i}$ quantities are weight factors and discrete velocities which in D2Q9 lattice, are given below :

$$
\omega_{i}=\left\{\begin{array}{l}
\frac{4}{9}(i=0) \\
\frac{1}{9}(i=1,2,3,4) \\
\frac{1}{36}(i=5,6,7,8)
\end{array}\right.
$$

$$
\left\{\begin{array}{l}
c_{0}=(0,0) \\
c_{2 i+1}=c\left[\cos \left(\frac{i \pi}{4}\right), \sin \left(\frac{i \pi}{4}\right)\right], i=0,1,2,3 \\
c_{2 i}=\sqrt{2}\left[\cos \left(\frac{(2 i-1) \pi}{4}\right), \sin \left(\frac{(2 i-1) \pi}{4}\right)\right], i=1,2,3,4
\end{array}\right.
$$

Where $c=\delta x / \delta t$. For simplicity we assume that $\delta x=\delta t=1$.

Chapman-Enskog expansion depicts that lattice Boltzmann equation satisfies the continuity and momentum equations.

With substituting equation (2) in equation (1) one obtains:

$$
\overline{f_{i}}(\vec{x}, t+\delta t)=f_{i}(\vec{x}, t)-\frac{1}{\tau}\left[f_{i}(\vec{x}, t)-f_{i}^{e q}(\vec{x}, t)\right]
$$

The above equation consists of two parts. The streaming term is given below.

$$
f_{i}\left(\vec{x}+c_{i} \delta t, t+\delta t\right)=\bar{f}_{i}(\vec{x}, t+\delta t)
$$

The equation (7) yields quantities of $f_{i}$ for adjacent areas after one moment and collision process is evaluated by equation (6). Fluid viscosity is given by relaxation time and the lattice sound velocity, as the following form:

$$
v=\left(\tau-\frac{1}{2}\right) c_{s}^{2}
$$

where $c_{s}=c / \sqrt{3}$.

For positive values of viscosity, it is necessary that the relaxation time parameter, $\tau$ be more than 0.5 . But stability conditions forces that this amount has to be enough larger than 0.5 . Macroscopic velocity and density in each point will be calculated by the following equations:

$$
\begin{gathered}
\rho=\sum_{i} f_{i}=\sum_{i} f_{i}^{e q} \\
\rho u=\sum_{i} c_{i} f_{i}=\sum_{i} c_{c} f_{i}^{e q}
\end{gathered}
$$

\section{Boundary Conditions}

In the present work, there are two kinds of boundary conditions. The first boundary condition is referred to the top of the cavity with uniform horizontal velocity (Fig1).

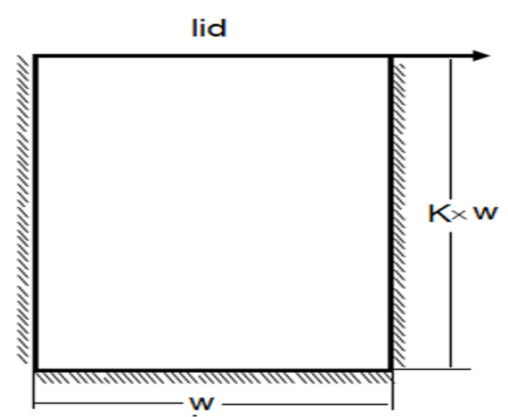

Figure 1. Schematic of lid driven cavity flow problem

Second boundary condition implies the static walls on the left, right and bottom of cavity. On the static walls no-slip boundary condition is applied and for this purpose bounce back method is used. On the other hand the orientation is fixed but direction is inversed. Pseudo-code of this method on the bottom wall is shown as follows: 


$$
\begin{aligned}
& f_{5}(x, 1)=f_{7}(x, 1) \\
& f_{2}(x, 1)=f_{4}(x, 1) \\
& f_{6}(x, 1)=f_{8}(x, 1)
\end{aligned}
$$

For the inlet boundary condition there are several suggestion such as Chen et al[15], Zou et al[2]. By these methods problem is easily solved when the aspect ratio $\mathrm{K}=1$, but by increasing the cavity depth, using the above methods cause the slow convergence. Hou proposed substitution of the equilibrium distribution function to distribution function in this way:

$$
f_{i}(x, N y)=f_{i}^{e q}(x, N y), \quad i=1, N x
$$

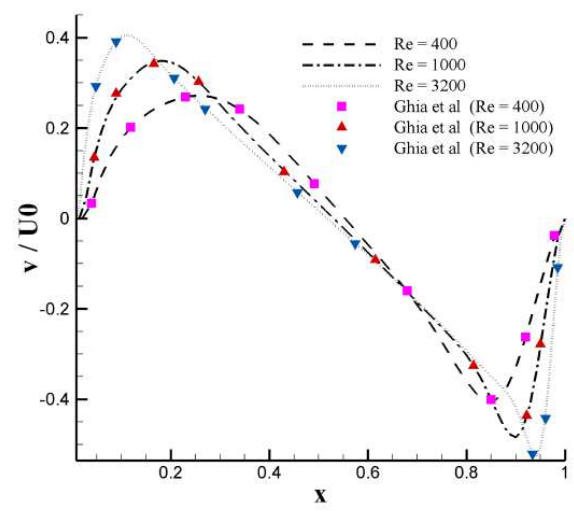

a

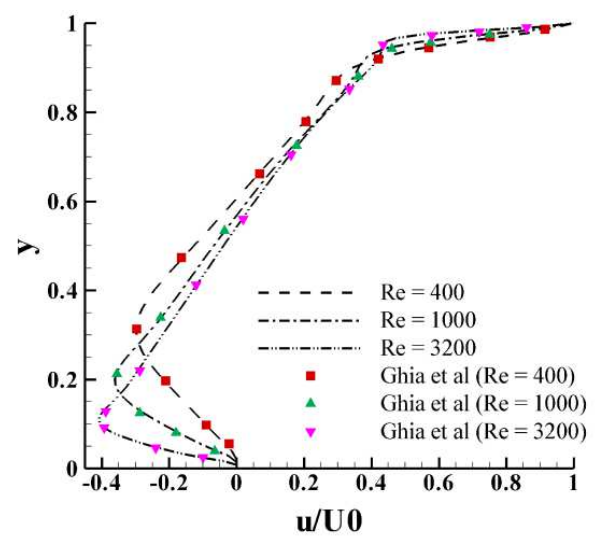

b

Figure 2. Comparisons of predicted (a) horizontal and (b) vertical velocity with Ghia et al. [8] at different Reynolds number-aspect ratio $K=1$

\section{Numerical Results}

Now the numerical solutions in case of various Reynolds numbers and aspect ratios in lid-driven cavity flow have been shown in figure. In this problem the dimensionless cavity Reynolds number is defined as $\operatorname{Re}=U_{0} N y / v$, where $U_{0}$ is uniform velocity which is on the top of the cavity, Ny is the width of the cavity and $v$ is the kinematic viscosity of fluid. Aspect ratio is characterized by $\mathrm{K}$ and its value is equal with $1,1.5$ and 4 respectively. In fixed $\mathrm{k}$ numbers we change the Re from 100 up to 5000. Lin et al[14] showed that mesh sizes have less effect on solutions accuracies especially when the mesh sizes are greater than $129 \times 129$.

To verify the written program code the comparisons of predicted (a) horizontal and (b) vertical velocity with Ghia et al.[8] at different Reynolds number of aspect ratio $\mathrm{K}=1$ are plotted in Fig.2 . For more precision the location of primary vortex and two bottom secondary vortices of $\mathrm{K}=1$ are shown in table 1 with solutions of[8], [18], [19], [20], [13] and[14]. Structural changes in the vortex at different Reynolds numbers of $\mathrm{K}=1$ are shown in Fig3.

To investigate the effect of cavity depth on flow structure, we will study the case of $\mathrm{K}=1.5$. Horizontal and vertical velocity at different Reynolds number at $\mathrm{K}=1.5$ are shown in Fig4. This figure presents the predicted horizontal and vertical velocities along $\mathrm{x}=0.5$ and $\mathrm{y}=0.75$, respectively. The appearance of the second primary vortex can be observed from Fig. 4a, where forward velocity is present at location for $y<0.2$. With increasing depth of the cavity, the vortices in the lower corners are growing.

As the Reynolds number is increasing these vortices merge and create second primary vortex (Fig.5). As the Reynolds number increases further, another two corner vortices would emerge. As we see this event (merger of corner vortices) did not happen in the previous case when $\mathrm{K}=1$. Comparing the detail results of the present work with Patil et al.[12], Pantil[13] and Lin et al.[14] are reported in table 2 .
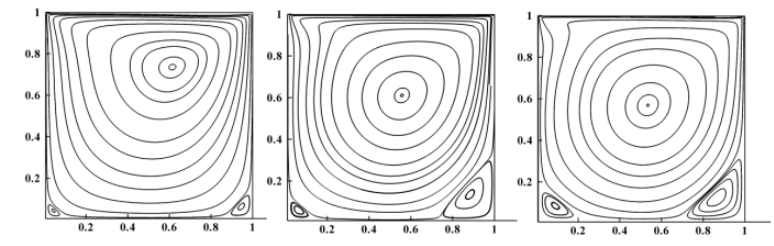

$\operatorname{Re}=100$

$\operatorname{Re}=400$

$\operatorname{Re}=1000$
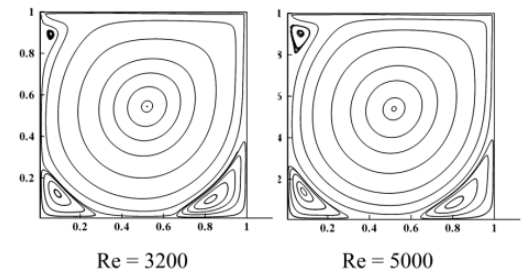

Figure 3. Streamline distributions at different Reynolds number-aspect ratio $K=1$. 


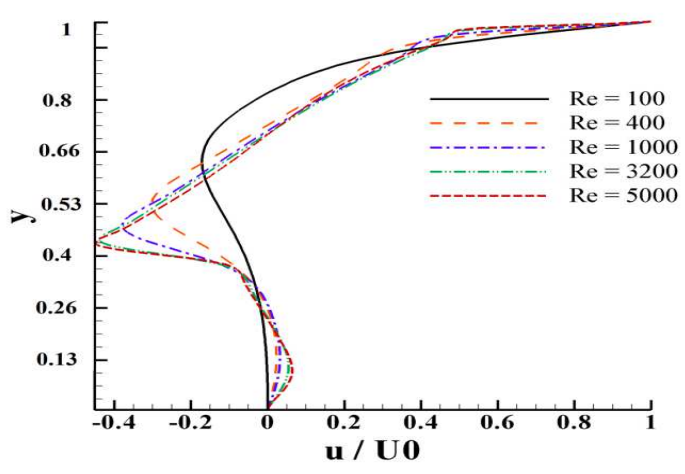

a

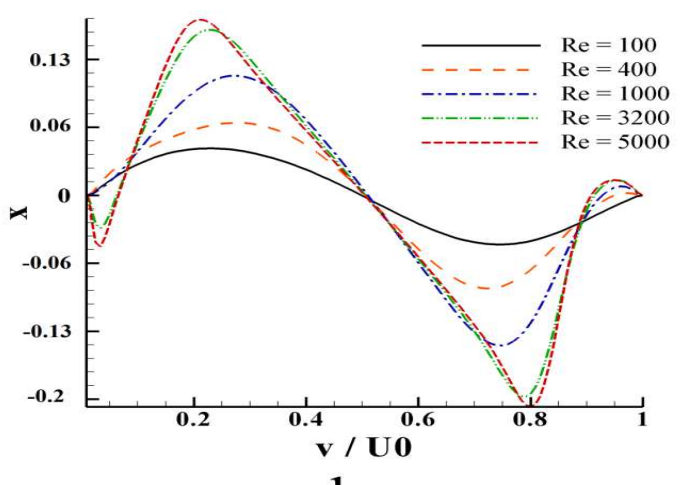

b

Figure 4. Predicted (a) horizontal and (b) vertical velocity at different Reynolds number, $K=1.5$

Table 1. Comparisons of the locations of the vortices at different Reynolds numbers with Ghia et al. [8], Pandit [13] and Lin et al[14] - aspect ratio K =1

\begin{tabular}{|c|c|c|c|c|c|c|}
\hline \multirow{2}{*}{$\operatorname{Re}$} & \multicolumn{2}{|c|}{ Primary vortex } & \multicolumn{2}{|c|}{ Left 2ndary vortex } & \multicolumn{2}{|c|}{ Right 2ndary vortex } \\
\hline & $\mathbf{x}$ & $\mathbf{y}$ & $\mathbf{x}$ & $\mathbf{y}$ & $\mathbf{x}$ & $\mathbf{y}$ \\
\hline 100 & & & & & & \\
\hline [8] & 0.7344 & 0.6172 & 0.0391 & 0.0313 & 0.0625 & 0.9453 \\
\hline [13] & 0.7273 & 0.6184 & 0.0439 & 0.0316 & 0.0575 & 0.9425 \\
\hline [14] & 0.7323 & 0.6140 & 0.0342 & 0.0346 & 0.0591 & 0.9448 \\
\hline $\begin{array}{c}\text { Current work } \\
400\end{array}$ & 0.7366 & 0.6179 & 0.0603 & 0.0606 & 0.0816 & 0.9416 \\
\hline$[8]$ & 0.6065 & 0.5547 & 0.0469 & 0.0508 & 0.1205 & 0.8906 \\
\hline [13] & 0.6065 & 0.5532 & 0.0439 & 0.0528 & 0.1384 & 0.8908 \\
\hline [14] & 0.6024 & 0.5543 & 0.0468 & 0.0510 & 0.1206 & 0.8875 \\
\hline $\begin{array}{c}\text { Current work } \\
1000\end{array}$ & 0.6133 & 0.5657 & 0.0659 & 0.0700 & 0.1439 & 0.8805 \\
\hline$[8]$ & 0.5625 & 0.5313 & 0.0781 & 0.0859 & 0.1094 & 0.8594 \\
\hline [13] & 0.5532 & 0.5266 & 0.0840 & 0.0840 & 0.1092 & 0.8577 \\
\hline [14] & 0.5645 & 0.5309 & 0.0776 & 0.0833 & 0.1117 & 0.8652 \\
\hline $\begin{array}{c}\text { Current work } \\
3200\end{array}$ & 0.5686 & 0.5346 & 0.0846 & 0.0904 & 0.1215 & 0.8646 \\
\hline$[8]$ & 0.5469 & 0.5165 & 0.1094 & 0.0859 & 0.0859 & 0.8125 \\
\hline [14] & 0.5396 & 0.5178 & 0.1195 & 0.0812 & 0.0843 & 0.8248 \\
\hline $\begin{array}{c}\text { Current work } \\
5000\end{array}$ & 0.5440 & 0.5203 & 0.1251 & 0.0909 & 0.0954 & 0.8253 \\
\hline$[8]$ & 0.5352 & 0.5117 & 0.1367 & 0.0703 & 0.0742 & 0.8086 \\
\hline [14] & 0.5349 & 0.5151 & 0.1365 & 0.0732 & 0.0730 & 0.8085 \\
\hline Current work & 0.5390 & 0.5176 & 0.1414 & 0.0837 & 0.0841 & 0.8055 \\
\hline
\end{tabular}

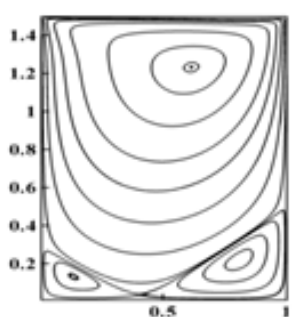

$\mathrm{Re}=100$

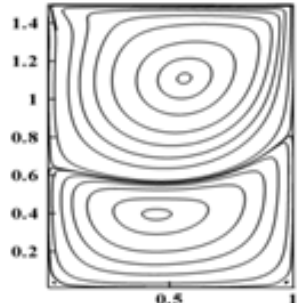

$\mathrm{Re}=400$

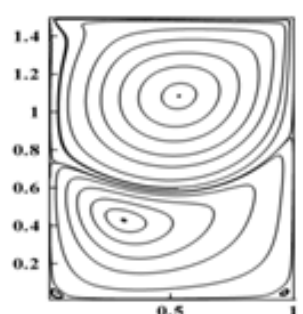

$\mathrm{Re}=1000$
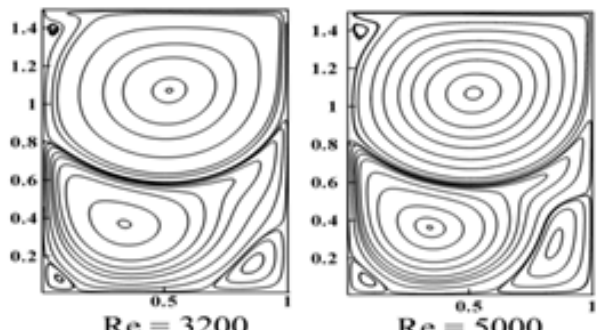

$\mathrm{Re}=3200$

$\mathrm{Re}=5000$

Figure 5. Variations of unsteady streamlines at one period-Re $=7500$ and aspect ratio $K=1.5$. 


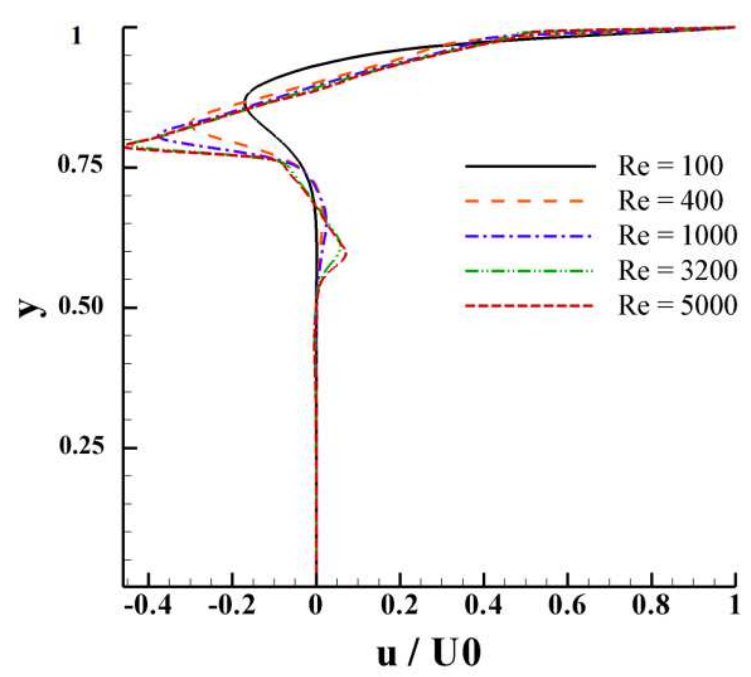

a

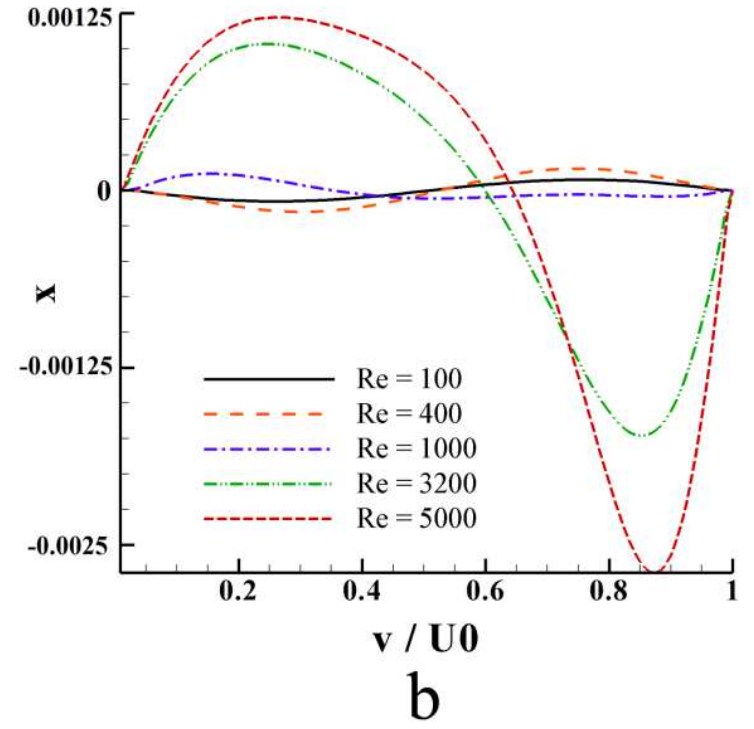

Figure 6. Predicted (a) horizontal and(b) vertical velocity at different Reynolds number-aspect ratio $K=4$

Fig. 6, showing the predicted horizontal and vertical velocities along $\mathrm{x}=0.5$ and $\mathrm{y}=2$, respectively. It can be seen that below $y=2$, the strength of the vortex is rather week, reflected by the low level of horizontal velocity. Streamlines in the case with $\mathrm{K}=4$ are shown in Fig.7. As we see in this figure with increasing depth to width ratio of the cavity and with growth of Re two bottom vortices are joining and create another primary vortex. This fact can be seen clearly in Fig.7 (a), (b) and (c). In the previous disquisitions and also in this paper, at $\mathrm{Re}=400$ three primary vortices was predicted while Pandit predicted four primary vortices which disagrees with Patil et al. , Lin et al. and current work. Patil also on his calculations at Reynolds numbers 1000 and 3200, predicted three primary vortices. The results of his work have been inconsistent with[13], [14, 17] and the present paper.

Comparisons of the locations of the primary vortices at different Reynolds numbers with Patil et al.[12], Pandit[13],Lin et al[14] and Navier-Stokes solutions are presented in table 3 .

\section{Conclusion}

In this paper LBGK model is used to simulate two dimensional lid-driven cavity flow[16]at various Reynolds numbers between 100 and 5000 and three aspect ratios, $\mathrm{K}=1$, 1.5 and 4 which is not used before. Implementation of appropriate boundary conditions is discussed in order to achieve reasonable convergence. It is reported that bounce back boundary condition in static walls with equilibrium distribution function in the inlet boundaries will tend to appropriate convergence. Flow structures have been studied in details and good agreements were obtained. For $\mathrm{K}=4$ cavity flow, four primary vortices are predicted by LBGK model for Reynolds number beyond 1000, which was not predicted by previous LBGK models, and results were verified by Lin et al.

Table 2. Comparisons of the locations of the vortices at different Reynolds numbers with Patil et al. [12] and Pandit [13] - aspect ratio $K=1.5$

\begin{tabular}{llccc}
\hline Re & \multicolumn{2}{l}{ 1st primary vortex } & \multicolumn{2}{l}{ 2nd primary vortex } \\
& $\mathbf{x}$ & $\mathbf{y}$ & $\mathbf{x}$ & $\mathbf{y}$ \\
\hline 400 & & & & \\
{$[12]$} & 1.1172 & 0.5625 & 0.3906 & 0.4453 \\
{$[13]$} & 1.1241 & 0.5399 & 0.3950 & 0.4205 \\
{$[14]$} & 1.1030 & 0.5522 & 0.3825 & 0.4259 \\
Current work & 1.1093 & 0.5596 & 0.3949 & 0.4468 \\
1000 & & & & \\
{$[12]$} & 1.0820 & 0.5352 & 0.4179 & 0.3007 \\
{$[13]$} & 1.0851 & 0.5399 & 0.3950 & 0.3439 \\
{$[14]$} & 1.0783 & 0.5293 & 0.4135 & 0.2960 \\
Current work & 1.0840 & 0.5346 & 0.4285 & 0.3111 \\
3200 & & & & \\
{$[12]$} & 1.0703 & 0.5195 & 0.3632 & 0.3320 \\
{$[14]$} & 1.0668 & 0.5175 & 0.3560 & 0.3293 \\
Current work & 1.0718 & 0.5208 & 0.3692 & 0.3394 \\
5000 & & & & \\
{$[14]$} & 1.0658 & 0.5151 & 0.3504 & 0.3322 \\
Current work & 1.0684 & 0.5178 & 0.3622 & 0.3411 \\
\hline
\end{tabular}




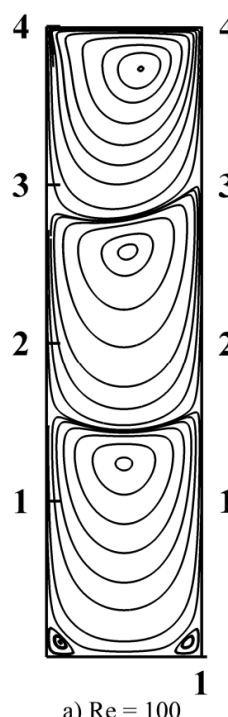

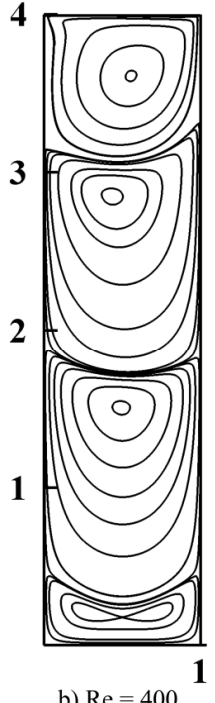

b) $\operatorname{Re}=400$

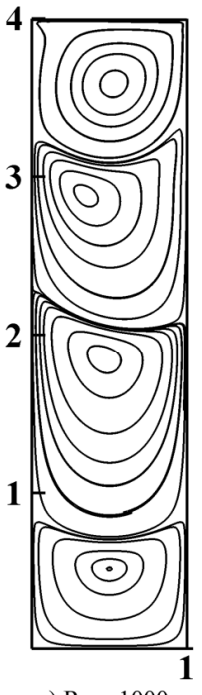

c) $\operatorname{Re}=1000$
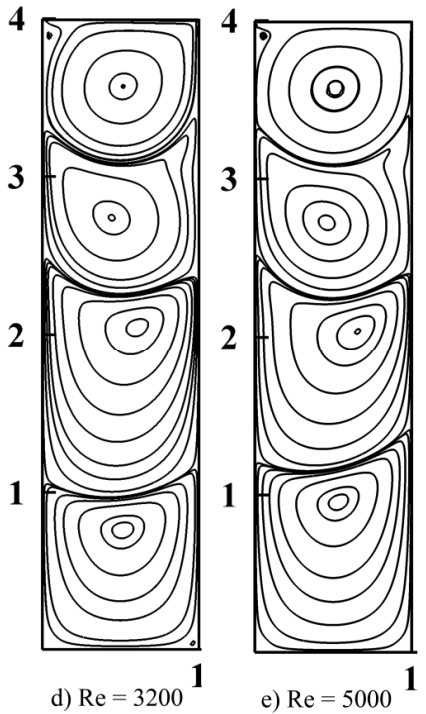

Figure 7. Streamline distributions at different Reynolds number-aspect ratio $K=4$

Table 3. Comparisons of the locations of the primary vortices at different Reynolds numbers with Patil et al. [12], Pandit [13],Lin et al[14] and Navier-Stokes solutions - aspect ratio $K=4$.

\begin{tabular}{|c|c|c|c|c|c|c|c|c|}
\hline \multirow{2}{*}{$\operatorname{Re}$} & \multicolumn{2}{|c|}{ 1st primary vortex } & \multicolumn{2}{|c|}{ 2nd primary vortex } & \multicolumn{2}{|c|}{ 3rd primary vortex } & \multicolumn{2}{|c|}{ 4th primary vortex } \\
\hline & $\mathbf{x}$ & $\mathbf{y}$ & $\mathbf{x}$ & $\mathbf{y}$ & $\mathbf{x}$ & $\mathbf{y}$ & $\mathbf{x}$ & $\mathbf{y}$ \\
\hline \multicolumn{9}{|l|}{$\overline{400}$} \\
\hline$[12]$ & 3.6172 & 0.5625 & 2.8515 & 0.4375 & 1.5000 & 0.5000 & - & \\
\hline [13] & 3.6029 & 0.5399 & 2.8750 & 0.4205 & 1.5266 & 0.5000 & 0.3300 & 0.5500 \\
\hline$[\mathrm{N}-\mathrm{S}]$ & 3.6078 & 0.5528 & 2.8439 & 0.4230 & 1.4875 & 0.4916 & - & \\
\hline$[14]$ & 3.6039 & 0.5528 & 2.8378 & 0.4250 & 1.4809 & 0.4914 & - & \\
\hline Current work & 3.6141 & 0.5638 & 2.8722 & 0.4472 & 1.5611 & 0.5033 & - & \\
\hline \multicolumn{9}{|l|}{1000} \\
\hline [12] & 3.5820 & 0.5352 & 2.8515 & 0.3437 & 1.7734 & 0.4179 & - & - \\
\hline [13] & 3.5834 & 0.5399 & 2.8750 & 0.3439 & 1.8801 & 0.4601 & 0.6667 & 0.5500 \\
\hline$[\mathrm{N}-\mathrm{S}]$ & 3.5794 & 0.5297 & 2.8361 & 0.3449 & 1.8244 & 0.4633 & 0.4459 & 0.5019 \\
\hline$[14]$ & 3.5778 & 0.5298 & 2.8356 & 0.3431 & 1.8167 & 0.4635 & 0.4385 & 0.5004 \\
\hline Current work & 3.5871 & 0.5386 & 2.8831 & 0.3574 & 1.8521 & 0.4759 & 0.5216 & 0.5100 \\
\hline \multicolumn{9}{|l|}{3200} \\
\hline [12] & 3.5703 & 0.5195 & 2.7226 & 0.4453 & 1.9961 & 0.5937 & - & \\
\hline [14] & 3.5648 & 0.5172 & 2.7132 & 0.4456 & 2.0090 & 0.6215 & 0.7247 & 0.5176 \\
\hline Current work & 3.5711 & 0.5212 & 2.7406 & 0.4488 & 2.0485 & 0.6134 & 0.7637 & 0.5196 \\
\hline \multicolumn{9}{|l|}{5000} \\
\hline [14] & 3.5650 & 0.5146 & 2.6996 & 0.4612 & 1.9900 & 0.6547 & 0.9628 & $0.5750 \quad 0.9553$ \\
\hline Current work & 3.5619 & 0.5168 & 2.7232 & 0.4637 & 2.0360 & 0.6593 & 0.5384 & \\
\hline
\end{tabular}

Sci Vol. 44 (2009), 137-155.

\section{References}

[1] Chen, S., Doolen, G.D., "Lattice Boltzmann method for fluid flow”, Ann Rev Fluid Mech, Vol. 30 (1998), 329-364.

[2] Zou, Q., He, X., "On pressure and velocity boundary conditions for the lattice Boltzmann BGK model", Phys Fluids, Vol. 9 (1997), 1591-1598.

[3] Niu, X.D., Shu, C., Chew, Y.T., "A thermal lattice Boltzmann model with diffuse scattering boundary condition for micro thermal flows", Comput Fluids, Vol. 36 (2006), 273-281.

[4] Ho, C.F., Chang, C., Lin, K.H., Lin, C.A., "Consistent boundary conditions for $2 \mathrm{D}$ and $3 \mathrm{D}$ laminar lattice Boltzmann Simulations", CMES-Comput Model in Eng \&
[5] Liu, C.H., Lin, K.H., Mai, H.C., Lin, C.A., "Thermal boundary conditions for thermal lattice Boltzmann simulations", Comput Math Appl Vol. 59 (2010), 2178-2193.

[6] Hou , S., Zou, Q., Chen, S., Doolen, G., Cogley, A.C., "Simulation of cavity flow by the lattice Boltzmann method", J Comput Phys, Vol. 118 (1995), 329-347.

[7] Guo, Z.L., Shi, B.C., Wang, N., "Lattice BGK model for incompressible Navier-Stokes equation", J Comput Phys, Vol. 165 (2000) 288-306.

[8] Ghia, U., Ghia, K.N., Shin, C.T., "High-Resolutions for incompressible flow using the Navier-tokes equations and a multigrid method", J Comput Phys, Vol. 48 (1982), 387-411.

[9] Erturk, E., Corke, T.C., Gökçöl, C., "Numerical Solutions of 
2-D Steady Incompressible Driven Cavity Flow at High Reynolds Numbers", Int. J. Numer. Meth. Fluids, Vol. 48 (2005), 747-774.

[10] Taneda, S., "Visulization of separating Stokes flows", Journal of the Physical Society of Japan, Vol. 46(6) (1979), 1935- 1941.

[11] Shen, C., Floryan, J.M., "Low Reynolds number flows over cavities", Phys Fluids, Vol. 28(11) (1985), 3191-3203.

[12] Patil, D., Lakshmisha, K., Rogg, B., "Lattice Boltzmann simulation of lid-driven flow in deep cavities", Comput Fluids, Vol. 35(10) (2006), 1116-1125.

[13] Pandit, S.K., "On the use of compact streamfunction-velocity formulation oflstready Navier-Stokes equations on geometries beyond rectangular", J Sci Comput, Vol. 36(2) (2008), 219-242.

[14] Lin, L.S., Chen, Y.C., Lin, C.A., "Multi relaxation time lattice Boltzmann simulations of deep lid driven cavity flows at different aspect ratios", Computers \& Fluids, Vol. 45(1) (2011), 233-240.
[15] Chen, S., Martinez, D., Mei, R., "On boundary conditions in lattice Boltzmann methods", Phys. Fluids, Vol. 8(9) (1996),2527-2537.

[16] JN Reddy : Applied functional analysis and variational methods in Engineering, Mc Graw Hill Book Company New York 1986.

[17] P Fischer, LW; Ho, GE; Karniadakis, EMR and Patera:Recent advanced in parellal spectral element simulation of unsteady incompressible fluid flows, computer and structures, 30, 217-231, 1988.

[18] D. Arumuga Perumal, Anoop K. Dass "Application of lattice Boltzmann method for incompressible viscous flows, Applied Mathematical Modeling vol. 14, 23-39, 2013.

[19] Reyad Omari "CFD simulation of Lid Driven cavity flow at moderate Reynolds Number, European Scientific Journal May 2013 edition vol.9 (15) 45-60.

[20] Anil Kumar , CL Varshney and Sajjan Lal “ Analytical study of effect of disorder on dispersionin steady inertial flows in porous effect, Scientific Research and Essays vol. 4(11) 1392-1402. 2009. 